\section{Optic disc cupping after optic neuritis evaluated with optic coherence tomography}

G Rebolleda ${ }^{1,2}$, S Noval', I Contreras ${ }^{1}$, F Arnalich-Montiel ${ }^{1}$, JL García-Perez ${ }^{1}$ and FJ Muñoz-Negrete ${ }^{1,2}$
${ }^{1}$ Ophthalmology

Department, Glaucoma Unit, Hospital Ramón y

Cajal, Madrid, Spain

${ }^{2}$ Surgery Department, Alcalá University, Alcalá de Henares, Spain

Correspondence:

G Rebolleda,

Ophthalmology

Department,

Glaucoma Unit,

Hospital Ramón y Cajal,

Carretera Colmenar Viejo

$\mathrm{km} \mathrm{9,} \mathrm{1,}$

Madrid E28034, Spain

Tel: + 34 913369023;

Fax: + 34913368126

E-mail: grebolleda@

telefonica.net

Received: 22 January 2008 Accepted in revised form: 29 March 2008

Published online: 25 April 2008

None of the authors has a commercial interest in any of the items mentioned in this study

\begin{abstract}
Purpose To determine whether the optic disc experiences cupping after an episode of optic neuritis as assessed by optical coherence tomography (OCT).

Methods A total of 50 patients with unilateral optic neuritis and 50 age- and sex-matched controls were studied. A complete examination, including visual acuity (VA), visual fields, and OCT scanning of the optic nerve head was performed. Mean cup-to-disc (C/D) ratios in the affected eyes were compared with fellow and control eyes. Results Mean C/D area ratio (CDAR), C/D vertical ratio (CDVR), and C/D horizontal ratio (CDHR) were significantly larger in the affected eyes compared to fellow $(P<0.001)$ and control eyes $(P<0.05)$. The asymmetry in CDAR, CDVR, and CDHR between both eyes in the patients with optic neuritis was equal to or greater than 0.2 in 24,28 , and $30 \%$ respectively. A significant inverse correlation was found between the $C / D$ ratios asymmetry and retinal nerve fibre layer (RNFL) thickness $(P<0.05)$. Conclusion A significant increase in $C / D$ ratio can be detected by OCT after unilateral optic neuritis, inversely correlated with RNFL thickness, and VA.

Eye (2009) 23, 890-894; doi:10.1038/eye.2008.117; published online 25 April 2008
\end{abstract}

Keywords: optic neuritis; optical coherence tomography; optic disc cupping; optic nerve head

\section{Introduction}

Even though optic disc cupping is usually identified with glaucoma, it may be seen in other, less common optic nerve diseases, such as congenital optic disc anomalies, ischaemic, hereditary and traumatic optic neuropathies, or in situations in which the anterior visual pathway is compromised, such as intracranial aneurysms or tumours. ${ }^{1-7}$ Although

traditionally it has been accepted that the most common change in the optic nerve head $(\mathrm{ONH})$ after optic neuritis is optic disc pallor, previous studies using optic disc photography have found that patients with optic neuritis have a larger cup in the affected eye compared with the fellow eye. ${ }^{8,9}$ Rath $e t \mathrm{al}^{8}$ described an increase in the cup-to-disc (C/D) ratio of 0.1-0.2 in the affected eye compared with the fellow eye; it was also greater than in a group of age-matched control subjects. In contrast, Radius and Maumenee ${ }^{10}$ found no significant increase in optic disc cupping. One drawback of these studies is that the value of the $\mathrm{C} / \mathrm{D}$ ratio depends greatly on the experience and subjective analysis of the evaluator. Trip et $a l^{11}$ using the Heidelberg Retina Tomograph (HRT) found an increase in the cup-shape measure after optic neuritis.

Optical coherence tomography (OCT) is an objective imaging technique that can obtain retinal images with a resolution of up to $10 \mu \mathrm{m} .{ }^{12,13}$ The repeatability of the $\mathrm{ONH}$ measurements with the Stratus OCT is moderately good, although OCT tends to provide higher $\mathrm{C} / \mathrm{D}$ ratios than those estimated by slit-lamp evaluation. ${ }^{14,15}$ The aim of this study is to determine whether optic neuritis leads to optic disc cupping using OCT to measure $\mathrm{C} / \mathrm{D}$ ratio in a group of patients with a single episode of unilateral optic neuritis and in a group of control subjects.

\section{Material and methods}

All patients with a single previous episode of unilateral optic neuritis that attended in the 
Department of Neuro-ophthalmology of our centre between 2000 and 2006 were identified through the review of hospital records and invited to participate in this study. The study was approved by the local ethic committee and informed consent was obtained from all subjects. The study adhered to the principles of the Declaration of Helsinki.

The diagnosis of optic neuritis was based on clinical criteria, including visual loss in the affected eye, the presence of a relative afferent pupillary defect, a visual-field defect, and pain that increases with eye movement. ${ }^{16}$ Inclusion criteria were the presence of an acute, unilateral episode of optic neuritis at least 6 months before the beginning of the study, no signs or symptoms of a previous episode in either eye, and age between 18 and 45 years. Exclusion criteria were the presence of an ocular pathology other than optic neuritis, intraocular pressure higher than $21 \mathrm{~mm} \mathrm{Hg}$ and a refractive error greater than $5.0 \mathrm{D}$ of spherical equivalent or 3.0 D of astigmatism in either eye.

All patients who met the study inclusion and exclusion criteria underwent a complete ophthalmologic evaluation including Snellen visual acuity (VA), biomicroscopy of the anterior and posterior segments (including clinical estimation of the vertical C/D ratio), funduscopy, intraocular pressure measurement, and visual-field testing on the same day as OCT imaging. Patients underwent Humphrey visual-field testing, using the Swedish Interactive Threshold Algorithm standard 24-2 strategy (Carl Zeiss Meditec, Dublin, CA).

OCT scanning was performed with the Stratus OCT (Carl Zeiss Meditec, Dublin, CA) after pharmacological mydriasis. Image acquisition was performed with the fast retinal nerve fibre layer (RNFL) thickness (3.46) and the fast optic disc protocols. Good-quality scans (signal score of 6 or higher) were saved as soon as they appeared. The RNFL thickness values were obtained with the OCT built-in software RNFL thickness average analysis protocol and the $\mathrm{ONH}$ parameters with the Optic Nerve Head Analysis protocol. Before recording these values, the examiner checked that the OCT had correctly identified the limits of the retinal pigment epithelium and manually reset them when necessary. The values recorded were the vertical disc diameter, the disc area, the C/D area ratio (CDAR), the C/D horizontal ratio $(\mathrm{CDHR})$, and the $\mathrm{C} / \mathrm{D}$ vertical ratio (CDVR).

Optical coherence tomography evaluation and a complete ophthalmological examination during the acute phase were available for 13 patients with retrobulbar neuritis. A separate, prospective analysis of these patients was performed, comparing initial $\mathrm{ONH}$ parameters with an evaluation performed 6 months after the episode.
Age- and sex-matched controls subjects were recruited from among the workers of our centre and the relatives of patients. Control subjects had no previous history of ocular diseases and no evidence of current pathology. They underwent the same ophthalmological evaluation as the patients. All the patients and the control subjects included in this study signed an inform consent approved by the Ethical Committee of the Institution.

Statistical analysis was performed using the SPSS 12.0 program for Windows (Inc., Chicago, USA). The Kolmogorov-Smirnov test was used to verify that the distribution of variable values was normal. A paired $t$-test was used to analyse ONH parameters and RNFL thickness differences between the affected and fellow eyes. An independent $t$-test was employed for comparisons with control subjects. The Pearson's correlation test was used to analyse the relationship between variables. The total level of significance was set to 0.05 .

\section{Results}

A total of 50 patients fulfilled the inclusion and exclusion criteria. Mean patient age at the time of the acute episode of optic neuritis was 33.6 years (standard deviation (SD) 5.9). The female:male ratio was $3: 2$. The mean time between optic neuritis and study inclusion was 2.4 years (SD 1.9). VA in the affected eye was 0.9 (SD 0.3) and 1.0 (SD 0.1) in the fellow eye. Visual-field mean deviation (MD) ranged from -1.2 to $-20.5 \mathrm{~dB}$ and -1.6 to $-4.9 \mathrm{~dB}$ respectively in the affected and fellow eyes.

Table 1 shows that, with no significant difference in $\mathrm{ONH}$ size, all C/D ratios were significantly higher in the affected eyes compared with the fellow eyes $(P<0.001)$. The mean difference in the $C / D$ ratios between the affected and fellow eyes was approximately 0.12. A difference equal to or greater than 0.2 between both eyes in CDAR, CDVR, and CDHR was found in 12 (24\%), 14 $(28 \%)$, and $15(30 \%)$ of patients respectively. Similarly, on slit-lamp evaluation mean vertical C/D ratio in the affected eye (0.46, SD 0.18) was also significantly higher

Table 1 Comparison of optic nerve head parameters between affected and fellow eyes

\begin{tabular}{lccc}
\hline & $\begin{array}{c}\text { Affected eyes } \\
\text { Mean }(S D)\end{array}$ & $\begin{array}{c}\text { Fellow eyes } \\
\text { Mean }(S D)\end{array}$ & P-value \\
\hline Disc area $\left(\mathrm{mm}^{2}\right)$ & $2.20(0.56)$ & $2.23(0.70)$ & 0.631 \\
Vertical disc diameter $(\mathrm{mm})$ & $1.90(0.27)$ & $1.95(0.29)$ & 0.739 \\
CDAR & $0.29(0.14)$ & $0.18(0.09)$ & 0.000 \\
CDVR & $0.51(0.13)$ & $0.39(0.11)$ & 0.000 \\
CDHR & $0.55(0.16)$ & $0.43(0.12)$ & 0.000 \\
\hline
\end{tabular}

Abbreviations: CDAR, cup-to-disc area ratio; CDHR, cup-to-disc horizontal ratio; CDVR, cup-to-disc vertical ratio. 
than in fellow eye (0.36, SD 0.15, $P=0.000)$. In total, 15 $(30 \%)$ patients had a difference equal to or greater than 0.2 in the estimated subjective vertical C/D ratio between both eyes.

Mean overall RNFL thickness was significantly thinner in affected eyes ( $81.89 \mu \mathrm{m}, \mathrm{SD} 22.6)$ compared with fellow eyes $(102.45 \mu \mathrm{m}, \mathrm{SD} 13.03, P=0.000)$. Mean overall RNFL thickness in the affected eyes was inversely correlated with VA $(r,-0.547, P<0.001)$. Mean C/D ratios in affected eyes were inversely correlated with overall RNFL thickness $(P<0.01)$ and with VA $(P<0.005)$ (data not shown). A significant inverse correlation was also found between C/D ratios asymmetry and RNFL thickness overall and by quadrant (Table 2).

To compare the characteristics of the $\mathrm{ONH}$ between patients with optic neuritis and the control group, one eye was randomly selected from each control subject (Table 3). There was a significant difference between affected and control eyes in CDAR, CDHR, CDVR, and mean RNFL thickness. The asymmetry in $\mathrm{C} / \mathrm{D}$ ratios was also significantly larger in patients than in controls $(P=0.0013)$. There were no significant differences between control and fellow patient eyes in the $\mathrm{ONH}$ parameters and mean RNFL thickness.

At the time of the acute optic neuritis, 26 (52\%) of patients had brain magnetic resonance imaging (MRI) changes consistent with demyelinative lesions. There

Table 2 Pearson's correlation between C/D ratio asymmetry and RNFL thickness overall and by quadrant

\begin{tabular}{lccccccc}
\hline & \multicolumn{2}{c}{$\begin{array}{c}\text { Mean } \text { CDAR } \\
\text { difference }\end{array}$} & \multicolumn{2}{c}{$\begin{array}{c}\text { Mean } \text { CDVR } \\
\text { difference }\end{array}$} & \multicolumn{2}{c}{$\begin{array}{c}\text { Mean } \text { CDHR } \\
\text { difference }\end{array}$} \\
\cline { 2 - 8 } & $\mathrm{r}$ & P-value & $\mathrm{r}$ & P-value & $\mathrm{r}$ & P-value \\
\hline Overall & -0.470 & 0.001 & -0.482 & 0.001 & -0.377 & 0.013 \\
Superior & -0.533 & 0.000 & -0.522 & 0.000 & -0.451 & 0.003 \\
Inferior & -0.438 & 0.003 & -0.451 & 0.002 & -0.374 & 0.015 \\
Nasal & -0.396 & 0.07 & -0.312 & 0.037 & -0.314 & 0.043 \\
Temporal & -0.538 & 0.000 & -0.377 & 0.011 & -0.415 & 0.006 \\
\hline
\end{tabular}

Abbreviations: CDAR, cup-to-disc area ratio; CDHR, cup-to-disc horizontal ratio; CDVR, cup-to-disc vertical ratio. were no significant differences between patients with and without lesions on MRI in the mean RNFL thickness of the affected eye $(86.36 \pm 19.6$ and $87.3 \pm 24.3$ respectively) $(P=0.267)$ and of the fellow eye (100.17 \pm 13.5 and $106.41 \pm 12.7$ respectively) $(P=0.121)$. No significant relationship was found between mean CDAR, CDHR, and CDVR asymmetry and the presence of lesions on MRI $(P=0.753, P=0.852$, and $P=0.594$ respectively).

There was no significant difference between patients with and without lesions on MRI in the visual-field MD of the fellow eye $(-1.6 \pm 1.5$ and $-0.7 \pm 1.4 \mathrm{~dB}$ respectively) $(P=0.056)$.

In the 13 patients with retrobulbar optic neuritis, 6 months after optic neuritis, there was a significant increase in $\operatorname{CDAR}(P=0.004), \operatorname{CDVR}(P=0.013)$, and CDHR $(P=0.008)$ in the affected eyes, with no significant change in the fellow eyes (Table 4).

Table 3 Comparison of optic nerve head parameters and mean RNFL thickness between affected, fellow, and control eyes

\begin{tabular}{|c|c|c|c|}
\hline & $\begin{array}{l}\text { Affected eye } \\
(\mathrm{n}=50)\end{array}$ & $\begin{array}{l}\text { Control eye } \\
(\mathrm{n}=50)\end{array}$ & $\begin{array}{l}\text { Fellow eye } \\
(\mathrm{n}=50)\end{array}$ \\
\hline Disc area $\left(\mathrm{mm}^{2}\right)$ & $2.20(0.56)$ & $2.26(0.47)$ & $2.23(0.70)$ \\
\hline$P$ & 0.200 & & 0.232 \\
\hline $\begin{array}{l}\text { Disc diameter } \\
(\mathrm{mm})\end{array}$ & $1.90(0.27)$ & $1.94(0.28)$ & $1.95(0.29)$ \\
\hline$P$ & 0.770 & & 0.716 \\
\hline CDAR & $0.29(0.14)$ & $0.22(0.15)$ & $0.18(0.09)$ \\
\hline$P$ & 0.048 & & 0.121 \\
\hline CDVR & $0.51(0.13)$ & $0.40(0.17)$ & $0.39(0.11)$ \\
\hline$P$ & 0.018 & & 0.245 \\
\hline CDHR & $0.55(0.16)$ & $0.42(0.11)$ & $0.43(0.12)$ \\
\hline$P$ & 0.000 & & 0.604 \\
\hline $\begin{array}{l}\text { RNFL thickness } \\
(\mu \mathrm{m})\end{array}$ & $81.89(22.60)$ & 104.51 (10.39) & 102.45 (13.03) \\
\hline$P$ & 0.000 & & 0.419 \\
\hline
\end{tabular}

Abbreviations: CDAR, cup-to-disc area ratio; CDHR, cup-to-disc horizontal ratio; CDVR, cup-to-disc vertical ratio; RNFL, retinal nerve fibre layer.

Values are expressed as mean (standard deviation).

Table 4 Cup-to-disc ratio change in eyes with retrobulbar optic neuritis

\begin{tabular}{|c|c|c|c|c|c|c|}
\hline & \multicolumn{2}{|c|}{$C D A R$} & \multicolumn{2}{|c|}{$C D V R$} & \multicolumn{2}{|c|}{ CDHR } \\
\hline & Acute & 6 months & Acute & 6 months & Acute & 6 months \\
\hline Affected eye & $0.22(0.16)$ & $0.31(0.20)$ & $0.42(0.17)$ & $0.50(0.17)$ & $0.47(0.19)$ & $0.58(0.20)$ \\
\hline$P$ & \multicolumn{2}{|c|}{0.04} & \multicolumn{2}{|c|}{0.013} & \multicolumn{2}{|c|}{0.08} \\
\hline Fellow eye & $0.25(0.25)$ & $0.22(0.20)$ & $0.46(0.21)$ & $0.43(0.13)$ & $0.47(0.19)$ & $0.44(0.16)$ \\
\hline$P$ & \multicolumn{2}{|c|}{0.656} & \multicolumn{2}{|c|}{0.131} & \multicolumn{2}{|c|}{0.477} \\
\hline
\end{tabular}

Abbreviations: CDAR, cup-to-disc area ratio; CDHR, cup-to-disc horizontal ratio; CDVR, cup-to-disc vertical ratio.

Values are expressed as mean (standard deviation). 


\section{Discussion}

Optic disc cupping can result from multiple disorders, the most common being glaucomatous optic neuropathy. ${ }^{1-7}$ To the best of our knowledge, this is the first study to use OCT to determine if optic disc cupping occurs after optic neuritis.

In normal subjects, mean $\mathrm{C} / \mathrm{D}$ ratio is approximately 0.3 and the difference in C/D ratio between both eyes is less than 0.1 in up to $98 \%$ of cases. ${ }^{17-19}$ An increase in $\mathrm{C} / \mathrm{D}$ ratio in eyes following unilateral $\mathrm{ON}$ has been suggested by several authors, although they employed subjective methods to measure the C/D ratio. Rath et $a l^{8}$ found that after optic neuritis, the affected eyes had larger cups compared with the fellow eye. All patients had a C/D ratio greater than 0.3 in the affected eye compared with $7.1 \%$ of the fellow eyes; the $C / D$ ratio increase after optic neuritis was $0.1-0.2$ in the affected eye. Trobe $e t a l^{9}$ performed a study in which stereoscopic fundus photographs of patients with several neuroophthalmologic diseases were evaluated by experienced observers, determining whether there was optic disc cupping. Of the 14 eyes diagnosed with retrobulbar optic neuritis, $16 \%$ were classified as having cupping.

Trip et $a l^{11}$ using HRT, found that the cup-shape measure was greater in affected eyes compared to control eyes. The cup-shape measure quantifies the three dimensional shape of the optic disc, giving an indication of the overall shape of the cup, but has no units.

In this study, we selected unilateral cases of optic neuritis to compare $\mathrm{ONH}$ parameters between the affected and fellow eyes. Mean CDAR, CDVR, and CDHR as measured by OCT were significantly greater in affected eyes compared to fellow eyes (Table 1) and the difference was equal to or greater than 0.2 in 24, 28, and $30 \%$ of patients (Figure 1). There were no significant differences in the disc diameter or disc area between both eyes that could have been responsible for a difference in $\mathrm{C} / \mathrm{D}$ ratio or have interfered with the $\mathrm{C} / \mathrm{D}$ ratio evaluation.

Recently, several studies have focused on the measurement of RNFL thickness after optic neuritis and multiple sclerosis (MS). RNFL thickness may predict visual recovery and lower RNFL values correlate with impaired visual function. ${ }^{20-26}$ These studies have also documented axonal loss in MS optic neuritis eyes, unaffected fellow eyes of patients with MS optic neuritis and MS without history of optic neuritis, suggesting that OCT is a promising tool to detect subclinical changes in RNFL. ${ }^{25,26}$ Thus, it is reasonable to suppose that in our study, there also might be some cupping in the fellow eye; therefore, we also included a group of control patients. No significant differences in $\mathrm{ONH}$ parameters and RNFL thickness $(P=0.419)$ were detected between fellow and control eyes (Table 3).

In the current study, approximately $50 \%$ of patients had cranial MRI lesions. Although patients with lesions on MRI had a thinner mean RNFL thickness in the fellow eye than those without MRI lesions, the difference was not statistically significant $(P=0.121)$. Moreover, the presence of lesions on MRI was not significantly related to differences in C/D ratios between affected and fellow eyes.

The mean visual-field MD of the fellow eye in patients having MRI lesions was slightly worse than in those having no lesions, but the difference was not significant $(P=0.056)$.

Data were available from 13 eyes with retrobulbar optic neuritis during the acute episode and up to 6 months later. A significant increase in $\mathrm{C} / \mathrm{D}$ ratios occurred only in the affected eyes (Table 4). This strengthens the hypothesis that the difference in $\mathrm{C} / \mathrm{D}$ ratio is really due to optic neuritis and not by chance.
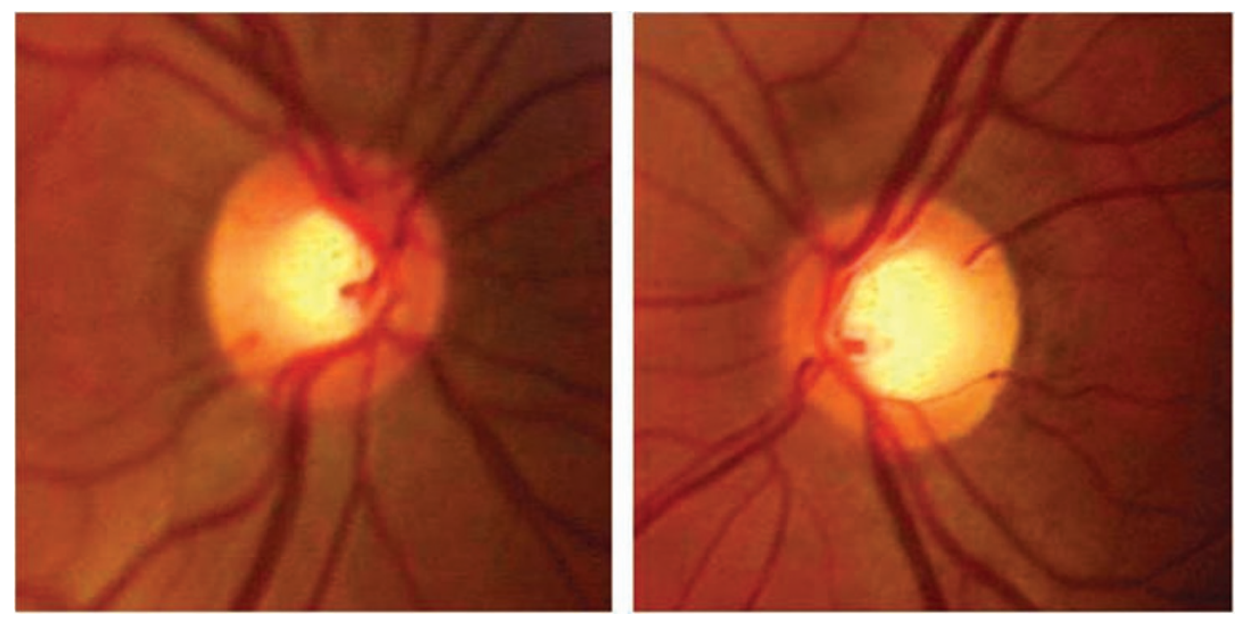

Figure 1 Marked asymmetry in cup-to-disc (C/D) ratio in left eye after optic nerve (ON) compared with normal right eye. 
A small increase in the $C / D$ ratio can be expected when the optic nerve loses axons in response to various noxious stimuli. In optic neuritis, optic disc cupping may be explained by the loss of retinal nerve fibres that has been documented by several authors. Previous studies found that OCT can detect axonal damage as early as the third month after an isolated episode of $\mathrm{ON}$ even in the presence of normal visual fields. ${ }^{20-24}$

In fact, in the current study, mean overall RNFL thickness was significantly lower in affected eyes compared with fellow eyes. Unsurprisingly, mean C/D ratios in affected eyes and $\mathrm{C} / \mathrm{D}$ ratios asymmetry were inversely correlated with mean overall RNFL thickness and VA.

Accordingly, in a process such as non-arteritic anterior ischaemic optic neuropathy, in which the ischaemic insult to the optic nerve leads to more intense damage than after optic neuritis, our group recently found that the CDAR increased $50 \%$ compared with the fellow eye. ${ }^{27}$

In the current study, we have documented with OCT the presence of disc cup enlargement after a single episode of unilateral optic neuritis. Affected eyes showed a small but significant increase of the optic disc C/D ratios compared with the fellow and control eyes. Optic disc cupping is larger in eyes having lower RNFL thickness and worse VA after optic neuritis. This leads us to support that optic neuritis should be included among the diseases that may cause non-glaucomatous optic disc cupping, mainly in cases with worse recovery.

\section{References}

1 Trobe JD, Glasser JS, Cassady J, Herschler J, Anderson DR. Nonglaucomatous excavation of the optic disc. Arch Ophthalmol 1980; 98: 1040-1050.

2 Gittinger Jr JW, Miller NR, Keltner JL, Burde RM. Glaucomatous cupping-sine glaucoma. Surv Ophthalmol 1981; 25: 383-390.

3 Bianchi-Marzoli S, Rizzo III JF, Brancato R, Lessel S. Quantitative analysis of optic disc cupping in compressive optic neuropathy. Ophthalmology 1995; 102: 436-440.

4 Gutman I, Melamed S, Ashkenazi I, Blumenthal M. Optic nerve compression by carotid arteries in low-tension glaucoma. Graefes Arch Clin Exp Ophthalmol 1993; 231: 711-717.

5 Greenfield DS. Glaucomatous versus nonglaucomatous optic disc cupping: clinical differentiation. Semin Ophthalmol 1999; 14: 95-108.

6 Ambati BK, Rizzo JF. Nonglaucomatous cupping of the optic disc. Int Ophthalmol Clin 2001; 41: 139-149.

7 Piette SD, Sergott RC. Pathological optic-disc cupping. Curr Opin Ophthalmol 2006; 17: 1-6.

8 Rath EZ, Rehany U, Linn S, Rumelt S. Correlation between optic disc atrophy and aetiology: anterior ischaemic optic neuropathy vs optic neuritis. Eye 2003; 17: 1019-1024.

9 Trobe JD, Glaser JS, Cassady J. Optic atrophy. Differential diagnosis by fundus examination alone. Arch Ophthalmol 1980; 98: 1040-1045.
10 Radius RL, Maumenee AE. Optic atrophy and glaucomatous cupping. Am J Ophthalmol 1978; 85: 145-153.

11 Trip SA, Schlottmann PG, Jones SJ, Garway-Heath DF, Thompson AJ, Plant GT et al. Quantification of optic nerve head topography in optic neuritis: a pilot study. $\mathrm{Br}$ J Ophthalmol 2006; 90: 1128-1131.

12 Huang D, Swanson EA, Lin CP, Schuman JS, Stinson WG, Chang W et al. Optical coherence tomography. Science 1991; 254: 1178-1181.

13 Hee MR, Izatt JA, Swanson EA, Huang D, Schuman JS, Lin $\mathrm{CP}$ et al. Optical coherence tomography of the human retina. Arch Ophthalmol 1995; 113: 325-332.

14 Arnalich-Montiel F, Muñoz-Negrete FJ, Rebolleda G, SalesSanz M, Cabarga C. Cup-to-disc ratio: agreement between slit-lamp indirect ophthalmoscopic estimation and stratus optical coherence tomography measurement. Eye 2007; 21: 1041-1049.

15 Neubauer AS, Krieglstein TR, Chryssafis C, Thiel M, Kampik A. Comparison of optical coherence tomography and fundus photography for measuring the optic disc size. Ophthalmic Physiol Opt 2006; 26: 13-18.

16 Smith CH. Optic neuritis. In: Miller NR, Newman NJ (eds). Walhs and Hoyt Clinical Neuro-ophthalmology. Vol 1, 6th ed. Lipincott Williams \& Willians: Philadelphia, USA, 2005, pp 293-347.

17 Carpel EF, Engstrom PF. The normal cup-disk ratio. Am J Ophthalmol 1981; 91: 588-597.

18 Jonas JB, Gusek GC, Naumann GOH. Optic disc, cup and neuroretinal rim size, configuration and correlations in normal eyes. Invest Ophthalmol Vis Sci 1988; 29: 1151-1158.

19 Varma R, Tielsch JM, Quigley HA, Hilton SC, Katz J, Spaeth GL et al. Race-, age-, gender-, and refractive error-related differences in the normal optic disc. Arch Ophthalmol 1994; 112: $1068-1076$.

20 Fisher JB, Jacobs DA, Markowitz CE, Galetta SL, Volpe NJ, Nano-Schiavi ML et al. Relation of visual function to retinal nerve fiber layer thickness in multiple sclerosis. Ophthalmology 2006; 113: 324-332.

21 Noval S, Contreras I, Rebolleda G, Muñoz-Negrete FJ. Optical coherence tomography in optic neuritis. Ophthalmology 2007; 114: 200.

22 Noval S, Contreras I, Rebolleda G, Muñoz-Negrete FJ. Optical coherence tomography versus automated perimetry for follow-up of optic neuritis. Acta Ophthalmol Scand 2006; 84: 790-794.

23 Trip SA, Schlottmann PG, Jones SJ, Altmann DR, GarwayHeath DF, Thompson AJ et al. Retinal nerve fiber layer axonal loss and visual dysfunction in optic neuritis. Ann Neurol 2005; 58: 383-391.

24 Costello F, Coupland S, Hodge W, Lorello GR, Koroluk J, Pan YI et al. Quantifying axonal loss after optic neuritis with optical coherence tomography. Ann Neurol 2006; 59: 963-969.

25 Sepulcre J, Murie-Fernandez M, Salinas-Alaman A, GarcíaLayana A, Bejarano B, Villoslada P et al. Diagnostic accuracy of retinal abnormalities in predicting disease activity in MS. Neurology 2007; 68: 1488-1494.

26 Pulicken M, Gordon-Lipkin E, Balcer LJ, Frohman E, Cutter G, Calabresi PA. Optical coherence tomography and disease subtype in multiple sclerosis. Neurology 2007; 69: 2085-2092.

27 Contreras I, Rebolleda G, Noval S, Muñoz-Negrete FJ. Optic disc evaluation by optical coherence tomography in nonarteritic anterior ischemic optic neuropathy. Invest Ophthalmol Vis Sci 2007; 48: 4087-4092. 